J. Lake Sci. (湖泊科学), 2019, 31(4): 1157-1168

DOI 10. 18307/2019. 0412

(c) 2019 by Journal of Lake Sciences

\title{
太湖湍流动能耗散率的廓线分布及其可能机制”
}

\author{
赵巧华 ${ }^{1}$, 刘 鹏 ${ }^{1}$, 陈纾杨 ${ }^{2}$, 周 妍 $^{1}$, 王健健 ${ }^{1}$,汪 靖 $^{1}$ \\ ( 1 : 南京信息工程大学水文气象学院,南京 210044) \\ (2: 苏州市气象局,苏州 215131)
}

\begin{abstract}
摘 要: 湍流不仅是导致物质、动量等在水一气交界面的交换、水柱内部输送的关键过程,也是促进浅水湖泊中底泥再悬 浮及水生生态系统演变的驱动力; 其中湍流动能耗散率 $(\varepsilon)$ 不仅是描述湍流动能变化的关键物理量, 也是刻画水体中湍 流产生机制的关键过程. 基于 2017 年 10 月 29 日- 11 月 2 日位于太湖梅梁湾采集的高频三维流速廓线和水温廓线观测 资料,结合东山气象站同期的风场和辐射等气象资料, 探讨太湖中 $\varepsilon$ 的分布特征及其变化的可能机制. 结果表明, $0.7 \mathrm{~m}$ 深 度以下水平方向上 $\varepsilon$ 介于 $10^{-8} \sim 10^{-7} \mathrm{~m}^{2} / \mathrm{s}^{3}$ 之间, 比垂直方向大一个数量级左右. 尽管大型浅水湖泊几何深度浅, 但其 $\varepsilon$ 的深度廓线依然存在典型的 3 层: $\varepsilon$ 随深度递减的风浪直接作用层, 深度从水面至 $1.0 \mathrm{~m}$ 左右; $\varepsilon$ 基本不随深度变化的常 数层,分布区间为水深 $1.0 \sim 1.9 \mathrm{~m}$; 随后是 $\varepsilon$ 随深度递减的底边界混合层. 热力分层的强弱 (垂向温差)、位置对常数层和 底部边界中 $\varepsilon$ 的贡献显著, 甚至造成 $\varepsilon$ 的常数层起始深度下移. 本研究有利于进一步理解大型浅水湖泊的动力学过程及 其对水生生态系统演变的驱动效应.
\end{abstract}

关键词: 湍流;湍流动能耗散率;风浪作用层;常数层;太湖

\section{Profile features of dissipation rates of turbulence in Lake Taihu and possible mechanism}

\author{
ZHAO Qiaohua ${ }^{1}$, LIU Peng ${ }^{1}$, CHEN Shuyang ${ }^{2}$, ZHOU Yan ${ }^{1}$, WANG Jianjian ${ }^{1} \&$ WANG Jing ${ }^{1}$ \\ (1: Hydro-meteorological School, Nanjing University of Information Science and Technology, Nanjing 210044, P.R.China) \\ (2: Suzhou Meteorological Bureau, Suzhou 215131, P.R.China)
}

\begin{abstract}
Turbulence is a key process regulating the exchange of matter and momentum not only across the water-gas interface, but also within water columns. It is also a driving force for the resuspension of sediments and the evolution of shallow lake ecosystems. The dissipation rate of the turbulent kinetic energy $(\varepsilon)$ is a key physical quantity which depicts the evolution of the turbulent kinetic energy, and discriminates turbulence production rate from mechanisms in water bodies. Based on observations for profiles of three-dimensional currents using Acoustic Doppler Current Profiles (ADCP) and records on water temperature and the wind field in Lake Taihu from October 29, 2017 to November 2, 2017, the profile features of $\varepsilon$ in this large-scale shallow lake were explored. The horizontal $\varepsilon$ is about $10^{-8}-10^{-7} \mathrm{~m}^{2} / \mathrm{s}^{3}$ below $0.7 \mathrm{~m}$ water depth, about one order of magnitude larger than the vertical $\varepsilon$. Despite the shallowness of the lake, there are still three typical layers of $\varepsilon$ for $u^{\prime}, v^{\prime}$, and $w^{\prime}$ : the wind-wave layer, where the water depth is shallower than $1.0 \mathrm{~m}$, exhibits that $\varepsilon$ decreases with depth due to wind forcing, Langmuir turbulence and wave breaking; constant layer, in the range of $1.0 \mathrm{~m}$ to $1.9 \mathrm{~m}$ or so, shows that $\varepsilon$ is little variation with the depth; bottom boundary mixed layer, below the constant layer, exhibits that $\varepsilon$ decreases with depth. In Lake Taihu, the strength and location of stratification significantly contribute to $\varepsilon$ in the constant layer and the bottom boundary, and even cause the initial depth of the constant layer of $\varepsilon$ to move downward. This study will help to further understand the kinetic process of large shallow lakes and their evolutionary effects on aquatic ecosystems.
\end{abstract}

Keywords: Turbulence; dissipation rate of the turbulent kinetic energy; wind-wave layer; constant layer; Lake Taihu

大型浅水湖泊 (太湖) 中, 动力过程不仅对其营养盐的内源释放 ${ }^{[-2]}$ 、沉积物再悬浮、水体浊度变化 ${ }^{[3-5]}$ 及 藻类的水平输移 ${ }^{[6]}$ 等有着重要作用, 而且对湖泊环境的变化、湖泊生态系统的演替有着深远和复杂的影

* 国家自然科学基金项目 (41371222,51609116) 和江苏省自然科学基金项目 (BK20160961) 联合资助. 2018-08-10 收稿;2018-12-17 收修改稿. 赵巧华(1972 ), 男, 博士, 教授;E-mail: qhzhao@ nuist.edu.cn. 
响 ${ }^{[7]}$. 由此诸多学者对太湖流场、波流共同作用对底泥再悬浮及浊度等的时空演变进行了深人研究 ${ }^{[8-10]}$, 揭 示了太湖风生流、补偿流等湖流结构及其对底泥起悬和悬浮物沉降的影响规律. 尽管该方面的研究取得了 长足的进展, 但多集中在悬浮物等水平输移、水平流场及其结构等方面, 而基本忽略了强度较弱 (通常较水 平运动低一个或一个以上的量级)却控制物质、热量垂向分布、输移的次生垂向运动. 由波浪破碎等动力作 用和热力作用产生的次生垂向运动控制着水气界面及水体内部的物质、能量及动量的交换和输送 ${ }^{[1]}$, 从而 导致其成为影响水生生态系统演变的重要物理过程 ${ }^{[12]}$.

湍流是次生垂向运动的重要组成部分. 其包含各种尺度的浴旋结构, 具有宽泛的时间尺度和空间尺度、 高度不稳定等特征; 且浴旋的拉伸、形变等影响着三维湍流的演变, 导致难以准确地定量描述各级湍流的产 生、演变及消亡的规律. 在描述湍流的参数中, 湍流动能耗散率 $(\varepsilon)$ 是度量湍流和混合程度的物理量 ${ }^{[13]}$ 、闭 合湍流动能方程的关键 ${ }^{[14]}$, 也是模拟湖泊、海洋等水体动力学及生物化学过程的前提. 近年随着声学多普 勒流速剖面仪 (Acoustic Doppler Current Profiles, ADCP) 的应用, 对海洋、近岸河口及海湾等处高频观测的三 维流速研究取得了重要进展: 基于能量波数谱在惯性子区间的特征, 成功地推求了 $\varepsilon^{[15]}$. 水平流速切变和浮 力作用导致的湍流各向异性可能对惯性子区间的波数范围造成影响, 进而影响 $\varepsilon$ 的计算误差 ${ }^{[16-17]}$; 初步揭 示了热力作用、波和流及其相互作用对 $\varepsilon$ 廓线的影响特征 ${ }^{[18-19]}$; 海底边界层中由流速切变产生的湍流动能 与耗散通常呈现局地平衡 ${ }^{[20]}$. 然而, 关于 $\varepsilon$ 的研究多数集中于海洋或深水湖泊, 而针对大型浅水湖泊中湍流 运动的研究却较为鲜见.

针对动力过程影响水生生态系统演变的研究中, 秦伯强等 ${ }^{[21]}$ 基于长期的野外观测和模拟实验提出了 “适度” 的湍流扰动促使蓝藻细胞通过碰撞形成的细胞团, 更容易在水动力消失后快速上浮形成水华; Zhou 等 ${ }^{[22]}$ 通过实验证实 “短时间尺度的湍流” ( 3 天左右) 有利于微囊藻的生长及其藻毒素的释放; 在富营养化 和春季温度偏高的前提下, 流速、流量等动力作用是制约汉江硅藻水华的关键 (敏感) 因子 ${ }^{[23-24]}$. 但有关内陆 湖泊中湍流的生态效应及其定量影响机制方面的研究相对贵乏, 基本仍处于 “定性” 的描述阶段. 即使在流 场数值模拟方面, 浴旋粘性系数、湍流垂直扩散系数多假定为常数 ${ }^{[8-10]}$, 从而导致降低了湖泊垂向运动的模 拟精度,限制了对蓝藻水华形成机理的认识及水生生态环境修复的效果.

鉴于分层与混合中的湍流运动对大型浅水湖泊的垂向结构、物质和能量输送及水生生态系统演变的作 用, 因而迫切需要研究、分析大型浅水湖泊中 $\varepsilon$ 的特征及其变化机制, 为探究大型浅水湖泊的动力过程、水 生生态系统中的物理、生物及化学过程的耦合效应奠定坚实的基础. 以太湖为研究对象, 利用 ADCP 和气象 场等观测资料, 探究大型浅水湖泊中 $\varepsilon$ 的廓线特征, 以期推动湖泊动力学及生态学相耦合的研究工作.

\section{1 资料与方法}

\section{1 观测资料}

太湖是典型的大型浅水湖泊, 面积为 $2338 \mathrm{~km}^{2}$, 平均水深不超过 $2 \mathrm{~m}$. 为了能持续观测及保证供电条件, 三维流速廓线、水温廓线及波浪的观测位置选择在中国科学院太湖湖泊生态系统研究站的栈桥 (图 1).

本文所用资料的观测时段为 2017 年 10 月 29 日 $02: 00$ 至 2017 年 11 月 02 日 23:30. 观测期间平均水深 为 $2.3 \mathrm{~m}$. 观测数据说明及处理方法如下:

采用美国 RoweTechnologies 公司生产的 ADCP (型号: Seaprofiler1200) 高频测定水体的三维流速廓线. 其 工作原理如下: 假定颗粒物的运动速度和水体流速一致, 当发射的声波经颗粒物散射后导致其声学多普勒 频移. 基于此频移与水体流速的关系推算水体三维流速的廓线分布. 与传统的流体流速测量方法相比, ADCP 具有高分辨率, 高精度等优点, 尤其适用于复杂环境下流体流速的测量. 高频观测 (频率 $4 \mathrm{~Hz}$ ) 三维流 速; 观测层厚为 $0.1 \mathrm{~m}$; 观测开始深度为 $0.69 \mathrm{~m}$; 观测层数 15 层. 针对原始三维流速数据, 采用 Turkey53H 的 方法 ${ }^{[25]}$ 进行奇异值的确定、剔除及插补.

利用英国 Valeport 公司生产的方向波浪仪 (型号: MIDAS) 观测波高、波周期等参数, 测定频率为 $8 \mathrm{~Hz}$, 每 $30 \mathrm{~min}$ 采样 4096 次 (约 $8 \mathrm{~min}$ 左右), 其观测结果为采样时段的统计值.

利用多个加拿大理查德布兰克研究公司 (RBR) 生产的水温仪 (型号: TR1060), 通过悬挂于不同深度测 定水温廓线, 深度间隔为 $10 \mathrm{~cm}$, 观测最大深度为 $1.9 \mathrm{~m}$; 时间间隔为 $10 \mathrm{~min}$, 即每隔 $10 \mathrm{~min}$ 测定 1 次水温. 

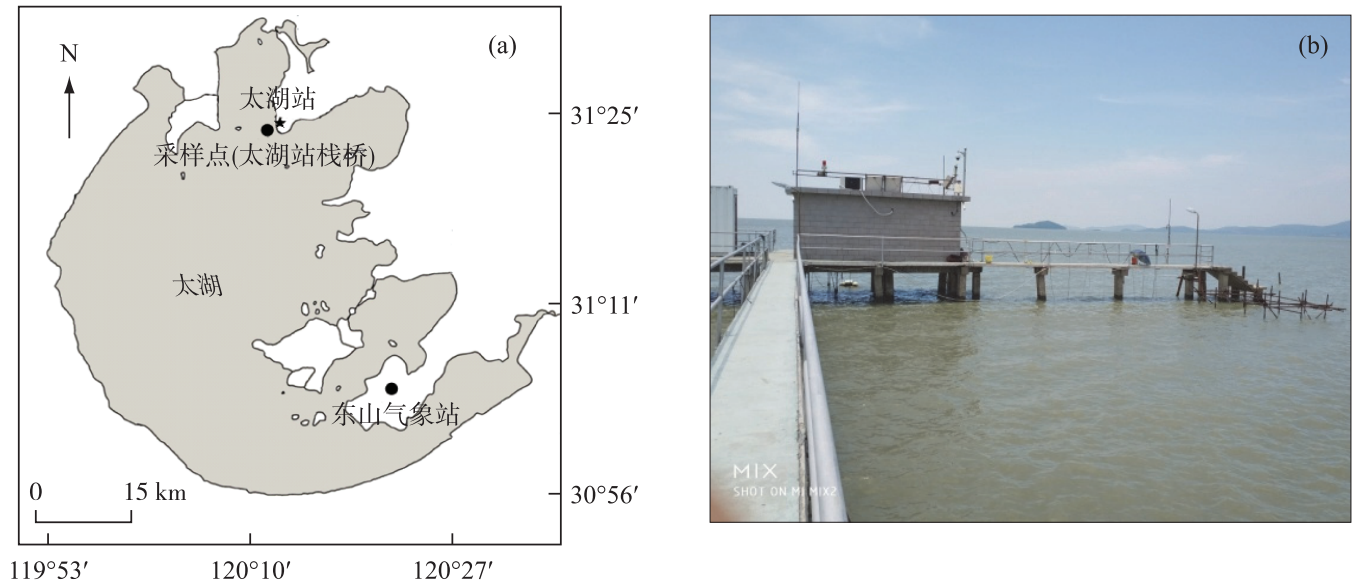

图 1 中国科学院太湖湖泊生态系统研究站位置 (a) 及栈桥 (b)

Fig.1 Location (a) and trestle bridge (b) of Ecosystem Observation and Research Station in Lake Taihu, Chinese Academy of Sciences

鉴于气象局的观测站点选址和业务观测相对规范, 时间连续性和空间代表性较好; 湖泊动力过程是大 气对水体驱动作用在空间、时间上的累积效应的综合反映, 而非局地 (空间尺度) 和瞬时 (时间尺度) 大气作 用的体现. 因而东山气象站 $\left(31.11^{\circ} \mathrm{N}, 120.43^{\circ} \mathrm{E}\right.$, 图 1) 的观测数据更具有空间代表性, 适用于探讨太湖水动 力过程. 本文采用苏州市东山气象站观测的风场 (仪器型号:ZQZ-TF; 安装高度 $10 \mathrm{~m}$ )、太阳短波辐射 (仪器 型号: WUSH-BRS; 安装高度 $1.2 \mathrm{~m}$ )、气压 (仪器型号: WUSH-TP300; 安装高度 $1 \mathrm{~m}$ )、相对湿度 (仪器型号: DHC2 ; 安装高度 $1.5 \mathrm{~m}$ )、云量 (定时人工观测)、气温(型号: WUSH-TW 100 ; 安装高度 $1.5 \mathrm{~m}$ ) 等数据. 风场的 观测频率均为逐小时, 辐射数据频率为 $1 \mathrm{~min}$.

\section{2 方法}

1.2.1 速度扰动量的分离 鉴于湖流是波一流相互作用的综合体现, 因而 $x 、 y 、 z$ 方向的瞬时流速 $(u 、 v 、 w)$ 包 含雷诺平均速度 $(\bar{u} 、 \bar{v} 、 \bar{w}) 、$ 波动轨迹速度 $(\tilde{u} 、 \tilde{v} 、 \widetilde{w})$ 、速度扰动量 $\left(u^{\prime} 、 v^{\prime} 、 w^{\prime}\right)$ 三个部分 ${ }^{[26] . ~}$

$$
\begin{aligned}
u & =\bar{u}+\tilde{u}+u^{\prime} \\
v & =\bar{v}+\tilde{v}+v^{\prime} \\
w & =\bar{w}+\widetilde{w}+w^{\prime}
\end{aligned}
$$

其中:

$$
\begin{aligned}
& \bar{u}=\frac{1}{N} \sum_{i=1}^{i=N} u_{i} \\
& \bar{v}=\frac{1}{N} \sum_{i=1}^{i=N} v_{i} \\
& \bar{w}=\frac{1}{N} \sum_{i=1}^{i=N} w_{i}
\end{aligned}
$$

式中, $N$ 是依据波浪仪在 $30 \mathrm{~min}$ 内测定的瞬时速度次数. 其中雷诺平均速度 $(\bar{u} 、 \bar{v} 、 \bar{w})$ 表示在观测时段内 (30 min 内) 瞬时速度的时间平均值, 其与时间无关, 即无论从时间序列中哪一时刻开始计算, 平均值都是相 等的; 位相平均速度 $(\langle u\rangle 、\langle v\rangle$ 、 $\langle w\rangle$ ) 是体现波一流耦合作用的瞬时速度样本的算术平均值, 其既 体现波浪的周期性、也是时间的函数,有别于雷诺平均.

$$
<u>=\frac{1}{M} \sum_{m=1}^{m=M} u(t+m \cdot T)
$$


波动轨迹速度产生于波浪作用,包含了波浪的周期信息, 体现了对时间的依赖性, 为位相平均速度与雷 诺平均速度之差 ${ }^{[26]}$ :

$$
\tilde{u}=\langle u>-\bar{u}
$$

式中, $T$ 为波浪周期, $m$ 为波浪的周期数, $M$ 为确定位相速度的总周期数. 为此可确定瞬时速度的扰动量 $\left(u^{\prime} 、 v^{\prime} 、 w^{\prime}\right)$.

1.2.2 湍流动能耗散率的计算 自然水体中的湍流动能主要来源于水平运动的垂直切变、波浪破碎、浮力及 对流等动力过程, 其中动能、势能 (或波能) 逐步衰减为各级湍浴, 最终通过粘性效应耗散. 可见, $\varepsilon$ 为单位时 间内由于分子粘性作用耗散单位质量水体中湍流动能的能力 ${ }^{[18]}$. 依据高频三维流速计算 $\varepsilon$ 的过程如下:

利用傅里叶变换,将速度扰动量 $\left(u^{\prime} 、 v^{\prime} 、 w^{\prime}\right)$ 转换成频率域 $\varphi(k), k$ 为波数,表达式如下:

$$
S^{2}=\overline{\left(\frac{\mathrm{d} \overrightarrow{u^{\prime}}}{\mathrm{d} z}\right)^{2}}=\frac{1}{\left(k_{\mathrm{U}}-k_{\mathrm{L}}\right)} \int_{k_{\mathrm{L}}}^{k_{\mathrm{i}}} \varphi(k)^{2} \mathrm{~d} k \approx \sum_{k_{\mathrm{L}}}^{k_{\mathrm{V}}} \varphi_{i}
$$

式中, $S$ 为速度扰动量的功率谱密度, 其波数的下边界 $k_{\mathrm{L}}$ 为 $2 \mathrm{cpm}$ (每分钟波的数量, count per minute), 上边 界 $k_{\mathrm{U}}$ 为 $10 \mathrm{cpm} . \overrightarrow{u^{\prime}}$ 表征速度扰动量 $\left(u^{\prime} 、 v^{\prime} 、 w^{\prime}\right)$ 的矢量.

迭代计算过程中, 设定最大波数 $\left(k_{\text {max }}\right)$ 为 $200 \mathrm{cpm}$, 波数步长为 $2 \mathrm{cpm}$. 迭代停止的条件有二:其一, 相近 两次计算的 Kolmogorov 波数 ( $k_{\mathrm{c}}$ ) 之差的绝对值不大于 $2 \mathrm{cpm}$; 其二, $k_{\mathrm{c}}$ 大于 $k_{\text {max }}$. 并基于公式 (10)、(11) 估 算 $\varepsilon^{[13]}$.

迭代计算确定波数的上下限 $(L, U)$,并计算区间 $[L, U]$ 的 $S^{2}$,进而依据下式计算 $\varepsilon$ :

$$
\varepsilon=7.5 v \overrightarrow{\left(\partial_{z} \overrightarrow{u^{\prime}}\right)^{2}}
$$

式中, $v$ 为动力粘性系数, 由此可通过公式 (11) 计算 $k_{\mathrm{c}}$, 即:

$$
k_{\mathrm{c}}=\left(\frac{\varepsilon}{v}\right)^{1 / 4}
$$

1.2.3 热量通量的计算 水一气之间的热量交换驱动水柱中水温的垂向分布, 进而通过水体分层、混合影响 湍流演变. 水一气之间的热量交换主要包括感热、潜热、长波辐射及短波辐射. 其中短波辐射为太阳辐射向 下传输, 因而气象观测站所获取的短波辐射基本可近似于水面上的短波辐射; 利用风场、气压、气温、短波辐 射、相对湿度、云量及表层水温等计算水体的感热、潜热、长波辐射, 由此结合太阳短波辐射计算水体获得的 净热量通量 $\left(R_{\mathrm{n}}\right)^{[27-30]}$. 计算过程简述如下:

水体获取的短波辐射通量 $\left(Q_{\mathrm{s}}\right)$ :

$$
Q_{\mathrm{s}}=Q_{\mathrm{s} 0}(1-\alpha)
$$

式中, $Q_{\mathrm{s} 0}$ 为下行短波辐射通量, $\alpha$ 为短波辐射的水面反照率.

水体获取的长波辐射通量 $\left(H_{\mathrm{LW}}\right)$ :

$$
H_{\mathrm{LW}}=\gamma \cdot \sigma \cdot T_{\mathrm{w}}^{4}\left(0.05 e_{\mathrm{a}}-0.39\right) B_{\mathrm{c}}-4 \gamma \cdot \sigma \cdot T_{\mathrm{w}}^{3}\left(T_{\mathrm{w}}-T_{\mathrm{a}}\right)
$$

式中, $\gamma$ 为水的长波发射率; $\sigma$ 为斯蒂芬波尔兹曼常数; $T_{\mathrm{a}}$ 和 $T_{\mathrm{w}}$ 分别为气温和表层水体的温度; $e_{\mathrm{a}}$ 为大气中的 水气压; $B_{\mathrm{c}}$ 为云量订正因子 $\left(B_{\mathrm{c}}=(1-0.72 C), C\right.$ 为云量 (成数) $)$.

水体获取的感热通量 $\left(H_{\mathrm{sen}}\right)$ :

$$
H_{\text {sen }}=\rho_{\mathrm{a}} \cdot \xi_{\mathrm{a}} \cdot U \cdot \kappa_{\mathrm{s}}\left(T_{\mathrm{w}}-T_{\mathrm{a}}\right)
$$

式中, $\rho_{\mathrm{a}}$ 为大气密度, $\xi_{\mathrm{a}}$ 为大气比热容, $\kappa_{\mathrm{s}}$ 为感热转换系数, $U$ 为风速.

水体获取的潜热通量 $\left(H_{\mathrm{Lat}}\right)$ :

$$
H_{\text {Lat }}=\rho_{\mathrm{a}} \cdot L_{\mathrm{e}} \cdot U \cdot \kappa_{\mathrm{l}}\left(q_{\mathrm{a}}-q_{\mathrm{sat}}\right)
$$

式中, $q_{\mathrm{a}}$ 为大气的比湿, $q_{\mathrm{sat}}$ 为饱和比湿, $\kappa_{1}$ 为潜热转换系数.

水体的净热量通量 $\left(R_{\mathrm{n}}\right)$ 为:

$$
R_{\mathrm{n}}=Q_{\mathrm{s}}+H_{\mathrm{LW}}+H_{\text {sen }}+H_{\text {Lat }}
$$

鉴于非常规气象站的观测要素通常难以满足热量通量计算的要求 (如云量的观测等), 因而选取满足计 算要求且几何距离相对较近的东山气象站获取的数据. 
1.2.4 Monin-Obukhov 长度计算 在相对稳定状态条件下, 风场驱动作用下的水柱表层中水平流速 $(\vec{U})$ 切变 湍流与 $\varepsilon$ 处于平衡状态 ${ }^{[26]}$. 并依据壁层定律计算水体摩擦速度 $\left(u_{*}\right)$, 即:

$$
\varepsilon(z)=\frac{u_{*}}{\kappa \cdot z}
$$

式中, $\kappa$ 为卡曼常数. 表层浮力通量 $\left(B_{o}\right)$ 的计算主要依据湖泊水体获取的净热量通量.

$$
B_{\mathrm{o}}=\frac{\alpha \cdot g \cdot R_{\mathrm{n}}}{\rho \cdot C_{\mathrm{p}}}
$$

式中, $\alpha$ 为水的热膨胀系数, $\rho$ 为水体密度, $C_{\mathrm{p}}$ 为水的比热. 则 Monin-Obukhov 长度 $(L)^{[18]}$ :

$$
L=-\frac{u_{*}^{3}}{\kappa \cdot B_{0}}
$$

式中, $L$ 表征了流场切变与浮力作用湍流产生率的比值, 刻画了两种作用对湍流动能产生的贡献比较.

\section{2 结果}

\section{1 气象场特征}

鉴于太湖的换水周期长 (300 天左右), 驱动水体分层与混合变化的机械动力主要来源于风场, 因而我 们给出了观测时段内的风速、风向特征(图 2). 从风速变化的整体趋势而言 (图 2a), 风速呈现递减的趋势; 从 10 月 29 日至 11 月 2 日, 每天均存在一个风速峰值, 但该峰值也随时间递减. 10 月 29 日和 10 月 30 日, 其 风速最大值为 $5.7 \mathrm{~m} / \mathrm{s}$, 后 3 天, 其峰值从 $3 \mathrm{~m} / \mathrm{s}$ 逐渐递减到 $2 \mathrm{~m} / \mathrm{s}$. 从对应的风向分布特征可见 (图 $2 \mathrm{~b}$ ), 4 天 内, 其风向频率最高的是东风, 占比为 $28 \%$, 其对应风速最大为 $3.5 \sim 4 \mathrm{~m} / \mathrm{s}$; 其次是东南或西北风, 各占比基 本均小于 $10 \%$, 总占比在 $40 \% \sim 50 \%$ 之间; 其中东南风风速最大达到 $5 \mathrm{~m} / \mathrm{s}$ 左右.
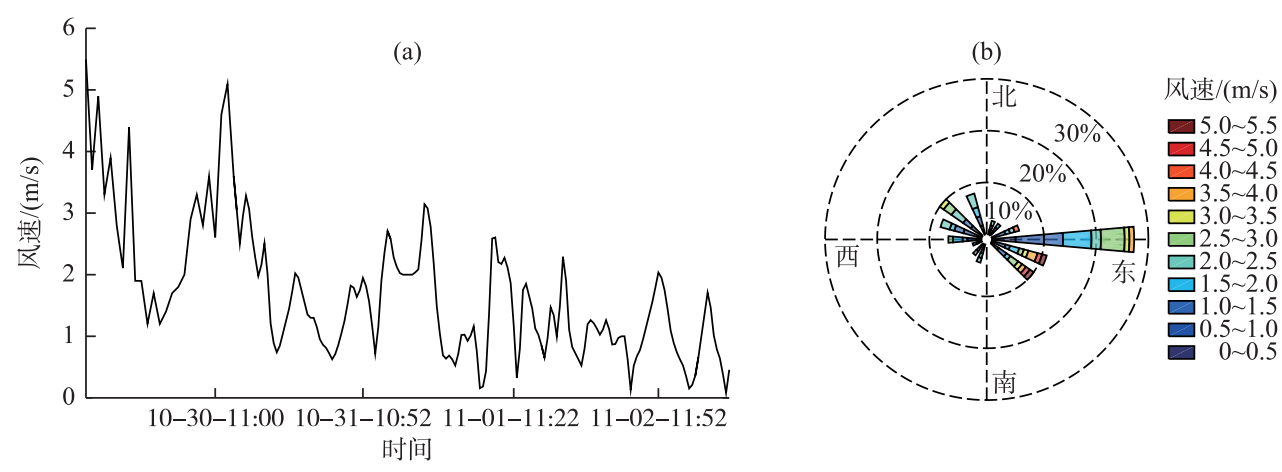

图 2 观测期间东山气象站风速的时序变化 (a) 以及风向玫瑰图 (b)

Fig.2 Temporal variation of wind speed (a) and wind direction rose (b)

at Dongshan Meteorological Station during observation period

驱动水体分层与混合演化的另一个作用来源于热力效应, 水一气之间的热量交换主要包括水体接受的 短波辐射、水一气之间的长波通量的交换、感热及潜热交换（图 3). 感热、潜热对水体获取或损失的净热量通 量影响甚微 (图 3), 进而也可说明不同气象站观测的风速差异导致的感热、潜热计算误差占 $R_{n}$ 的比重小; 水 体获取的净热量通量来源于太阳短波辐射, 水一气之间的长波交换主要表现为水体能量的损失; 从 10 月 30 日-11月 2 日,无论是太阳短波辐射还是净热量通量不仅呈现显著地日变化, 而且略呈递减的趋势; 晚间水 柱主要通过长波辐射的交换造成能量损失,且呈现弱的昼夜变化.

\section{2 水温及波浪场特征}

水柱的温度廓线不仅可以表征温度随时间的变化, 同时还能刻画水柱的热力结构, 是水体动力、热力作 用的综合体现. 由图 4 可见,水温基本呈现白天升高、晚上递减的周期性变化; 10 月 29、30 及 31 日水温基本 不随深度变化, 即水温上下基本一致, 完全混合; 11 月 1 日和 2 日上下温差超过 $1^{\circ} \mathrm{C}$, 存在明显的日分层现 


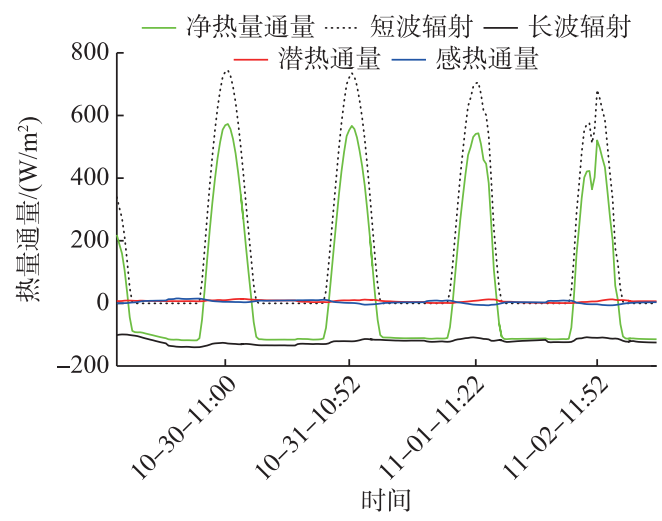

图 3 观测期间水一气热力交换通量的时序变化

Fig.3 Temporal changes of heat flux between water and air during observation period

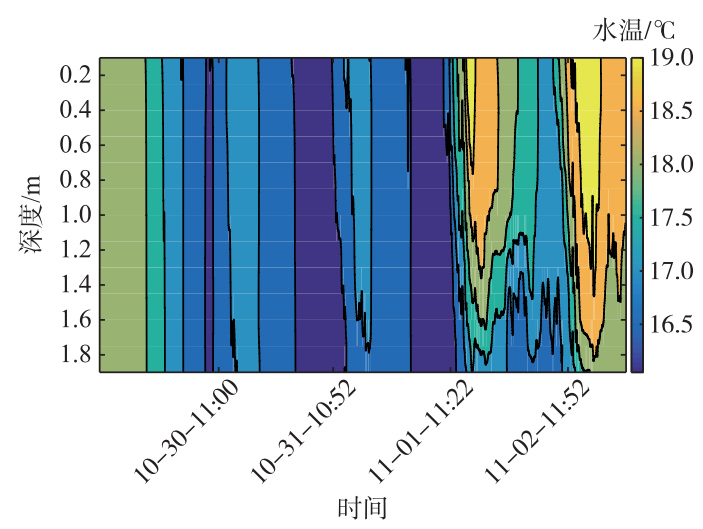

图 4 观测期间水温廓线的时序变化

Fig.4 Temporal variation of water temperature profiles during observation period

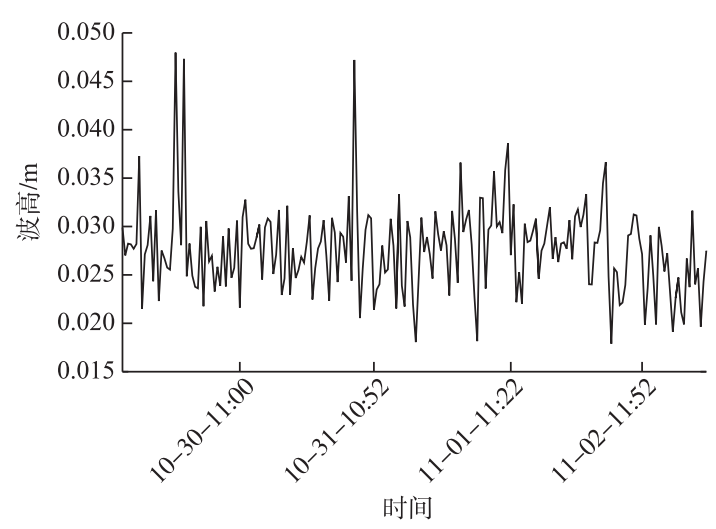

图 5 观测期间最大波高的时序变化

Fig.5 Time-varying of maximum wave heights during observation period
象. 11 月 2 日较 11 月 1 日的分层现象更明显且持 续时间也相对较长.

鉴于水体湍流产生与波浪关系密切 (波流相 互作用形成的朗缪尔环流、波浪破碎等), 因而本 文给出了观测时段最大波高的时间序列(图 5). 10 月 $29 、 30$ 及 31 日均存在较大的波高, 使得这 3 天 水体得以混合, 上下温差消失. 11 月 1 日、 2 日波 浪呈减弱的趋势, 尤其是 11 月 2 日减弱显著. 另, 鉴于观测位置并不位于太湖的开阔处, 导致在观 测时段中波高和风速的对应关系并不一致, 说明 风场、热力及波浪的耦合对水体动力影响存在显 著的空间分异.

\section{3 三维流速的 $\varepsilon$ 廓线分布}

基于 $\varepsilon$ 的物理意义,可知 $\varepsilon$ 可定量描述自然水 体中湍流、混合强度及衡量湍流动能演变过程. 图 $6 \mathrm{a}$ 给出了观测时段 $u 、 v 、 w$ 方向上 $\varepsilon$ 时间平均的深 度廓线. $\varepsilon$ 的平均值为 $10^{-7.58} \mathrm{~m}^{2} / \mathrm{s}^{3}$, 最大值出现在 表层, 最小值出现在 $2.1 \mathrm{~m}$; 在 $u 、 v$ 方向上的 $\varepsilon$ 基本 接近; $w$ 方向的 $\varepsilon$ 最小, 其值为 $10^{-9.14} \mathrm{~m}^{2} / \mathrm{s}^{3}$. 在 $0.7 \sim 1.0 \mathrm{~m}$ 深度区间, $\varepsilon$ 随深度递减; 在 $1.0 \sim 1.9 \mathrm{~m}$ 的深度区间, 其随深度基本呈现稳定状态, 即不随 深度变化; 在 1.9 2.1 m 区间, $\varepsilon$ 随深度递减.

鉴于热力作用对湍流动能存在产生与抑制的 两种作用, 因而在观测时段中, 以水体获取 (损失) 净热量通量为依据对观测时段的 $\varepsilon$ 进行平均 (图 $6 \mathrm{~b})$, 二者区别很小, 仅在 $w$ 方向的 $\varepsilon$ 廓线中部存 在稍明显的差别, 即在净热量通量小于零的时段 内 $\varepsilon$ 廓线的中部略大.

由于观测时段中含有完整的 4 天 (10 月 30 日、 10 月 31 日、 11 月 1 日和 11 月 2 日), 风速逐日 减小, 净热量通量也呈现出递减的趋势, 但递减趋 势较缓. 因此, 分别比较水平方向和垂直方向的 $\varepsilon$ 随日期的变化. 4 天内 3 个方向的 $\varepsilon$ 呈现类同的变 化特点: 其一, 上部随深度递减的特征基本不变; 在 11 月 1 日和 2 日出现了 $\varepsilon$ 随水深弱递增的现 象, 其中 11 月 2 日尤甚; 其二, 中部, 呈现随深度稳 定不变; 在后两天该深度区间的 $\varepsilon$ 有明显升高, 且 11 月 2 日升高程度最大; 后两天随深度维持不变 的深度区间较小; 其三, 底部, 后两天的 $\varepsilon$ 随深度递 减的趋势逐渐变弱.

\section{3 讨论}

自然水体中湍流动能主要来源于平均流速切 变、波浪动能、浮力效应导致的势能演变等过程, 
其中仅有浮力过程可能是湍流动能的源或汇 ${ }^{[18,31]}$, 另一方面, 湍流动能耗散是湍流动能生、消平衡和维持湍 流动能变率的关键物理过程,也是评估水体混合机制的关键物理量.

在 10 月 29 日至 11 月 2 日的观测时段, $0.7 \mathrm{~m}$ 深度以下 $u 、 v$ 方向的 $\varepsilon$ 介于 $10^{-8} \sim 10^{-7} \mathrm{~m}^{2} / \mathrm{s}^{3}$ 之间; $w$ 方向 的 $\varepsilon$ 数量级为 $10^{-9} \mathrm{~m}^{2} / \mathrm{s}^{3}$, 接近于 $\varepsilon$ 的背景值 ${ }^{[18]} \cdot \varepsilon$ 的风浪直接作用层、常数层及底边界摩擦混合层的分布特 征基本与 Callaghan 等 ${ }^{[18]}$ 的结果类似; 其中的唯一差别是 11 月 1 日、 2 日在风浪直接作用层与常数层之间的 过渡区 (图 6c、d、e).

\section{1 风浪强迫作用深度}

上层水体的湍流混合动力主要来源于风场的直接驱动、朗缪尔环流的效应及波浪破碎的作用 ${ }^{[18]}$. 其中 波浪破碎造成波能直接转化为湍流动能, 增强表层的 $\varepsilon$, 但 $\varepsilon$ 随深度衰减剧烈 ${ }^{[32]}$, 因而直接影响的深度一般 在几个波高范围之内 ${ }^{[31,33]}$. 鉴于本文采用悬挂式观测 , 无法观测到该浅表层湍流特征, 因而对此不作深人 讨论.

在受波浪破碎强烈影响的浅表层以下, 主要是由水平流速切变影响的壁层 ${ }^{[18,33]}$, 其特征是 $\varepsilon$ 随深度呈 反比; $\varepsilon$ 对深度的依赖弱于浅表层. 在本文 $\varepsilon$ 的廓线中 (图 6a), 各种条件下的 $\varepsilon$ 均存在随深度递减的特征 (图 6), 其深度基本在 $1.0 \mathrm{~m}$ 左右. 对应风速为 $0.2 \sim 5.7 \mathrm{~m} / \mathrm{s}$ (图 2); 有效波高基本小于 $10 \mathrm{~cm}$ (图 5). 可见, 在低中风速条件下, 风浪对水柱的直接作用深度为 $1.0 \mathrm{~m}$ 左右.

\section{$3.2 \varepsilon$ 的常数层中热力及朗缪尔环流效应}

在风浪直接作用深度以下至 $1.9 \mathrm{~m}, \varepsilon$ 的平均值随深度稳定少变, 即为常数层. 鉴于风场驱动的流速垂 直切变和波浪破碎等湍流产生作用随深度递减, 在 $\varepsilon$ 的常数层中, 必定存在湍流动能的产生与耗散的平衡, 且该湍流动能的产生不仅与深度相对独立, 而且其作用深度超过风浪直接作用的深度. 从产生湍流动能的 物理过程来看, 仅有对流作用符合常数层的要求. 其中对流产生的机制包含热力和动力两个方面: 其一, 水 体表面热量的损失 (感热、潜热和长波)导致的对流; 其二, 在表层混合层相对较浅的条件下,斯托克斯漂流 和风生流的相互作用可能触发朗缪尔环流 ${ }^{[32]}$. 通常朗缪尔环流存在于表层混合层的底部区间, 但在辐合区 朗缪尔环流效应产生的对流作用得以向下延伸 ${ }^{[18]}$. 海洋或深水湖泊中, 朗缪尔环流的影响深度远小于其几 何深度, 因而常数层中的 $\varepsilon$ 主要是热力作用引起的对流效应与之平衡 ${ }^{[18]}$; 但对浅水湖泊而言, 朗缪尔环流效 应产生的对流可能伸及底部边界层的顶部.

依据图 3 中净热量通量随时间的变化、图 6 中太湖在获取 (或失去) 能量条件下 $u 、 v 、 w$ 方向上 $\varepsilon$ 的时间 平均廓线,发现常数层中两种条件下 $u 、 v$ 方向的 $\varepsilon$ 基本相同, 仅在水体失去能量的条件下 $w$ 方向的 $\varepsilon$ 略大于 水体获得能量的 $\varepsilon$. 可见在水平方向上, 朗缪尔环流的对流输送效应可能占比较大, 造成热力效应引起的对 流影响基本可以忽略; 但在 $w$ 方向, 热力作用引起的对流湍流相对较强, 使得在水体失去能量条件下常数层 中 $w$ 方向的 $\varepsilon$ 相对偏大.

总之, 太湖中, 常数层中的 $\varepsilon$ 可能受到朗缪尔环流及热力效应共同引起的对流作用影响. 其中 $u 、 v$ 方向 上朗缪尔环流作用对常数层的 $\varepsilon$ 作用较大,而 $w$ 方向上热力效应的影响相对明显.

\section{3 动力和热力对 $\varepsilon$ 廓线的影响比较}

太湖水体深度浅, 水体储热能力相对较弱, 因而其对气象场的响应迅速, 造成水体分层、混合的时间尺 度一般为日, 甚至更短. 水体湍流动能产生来源于机械动力和热力作用. 其中机械动力主要包括风浪、朗缪 尔环流等过程; 热力学效应主要是通过水体获取或损失能量造成的分层、混合之间的转换,进而产生或抑制 湍流,另一方面,不同机制产生的湍流所涉及的深度存在差异 ${ }^{[34]}$.

鉴于图 6a、6b 两图中 $\varepsilon$ 廓线是所有观测时段的平均,因而掩盖了热力和朗缪尔环流对 $\varepsilon$ 影响的差异. 本 文观测时段包含了 4 整天,期间风速逐渐减小,净辐射通量的日变化程度相对较弱,因而通过 10 月 30 日、31 日、11月 1 日、11月 2 日的 $\varepsilon$ 廓线分布,可进一步区分风浪和热力作用对 $\varepsilon$ 廓线的影响.

4 天 $u 、 v 、 w$ 方向的 $\varepsilon$ 在风浪直接作用层中基本相同; 该时段中的后两天上下水温差超过 $1^{\circ} \mathrm{C}$ (图 4), 除 了风浪的直接作用, 对流混合对该层中 $\varepsilon$ 的影响在 4 天平均中并未得到显著体现 (图 6a). 为此, 依据水温的 垂向廓线分布特征和 $\varepsilon$ 的位置对温度进行插值, 得到在各 $\varepsilon$ 位置上、下 $5 \mathrm{~cm}$ 的水温, 并计算各 $\varepsilon$ 对应 $10 \mathrm{~cm}$ 深度区间的温差时间序列(图 7). 11 月 1 日、2 日, 风浪直接作用的深度区域, 温差远小于下部, 且每 $10 \mathrm{~cm}$ 

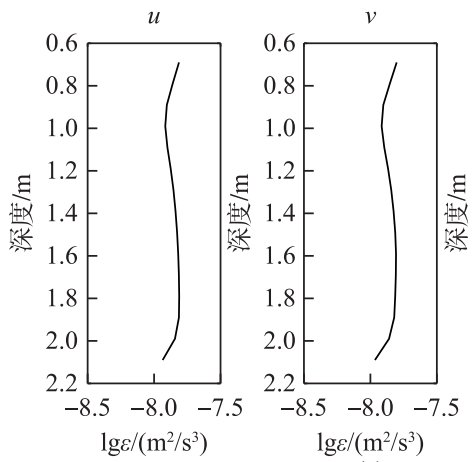

(a)
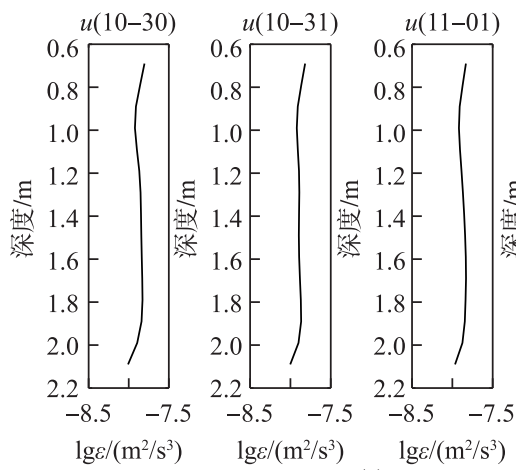

(c)
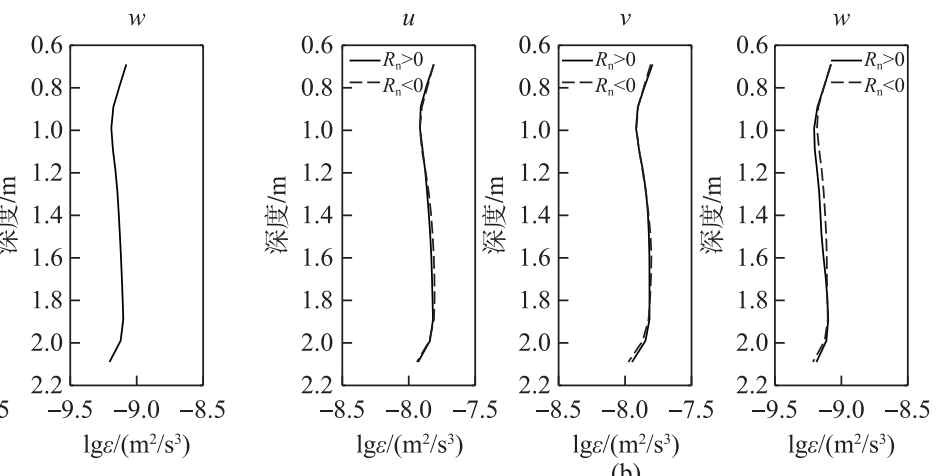

(b)
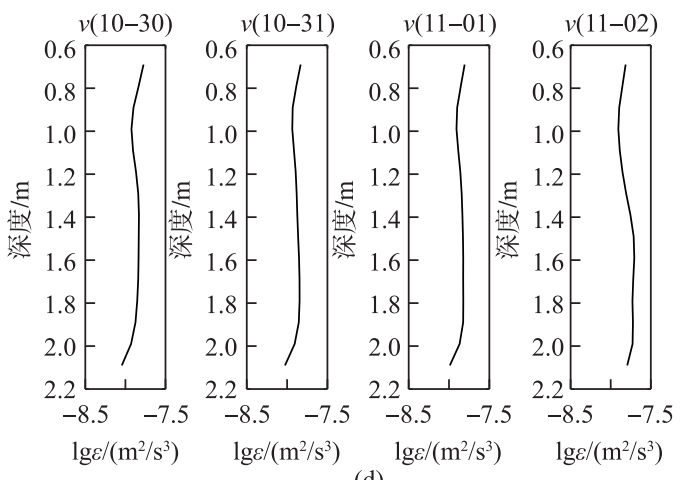

(d)

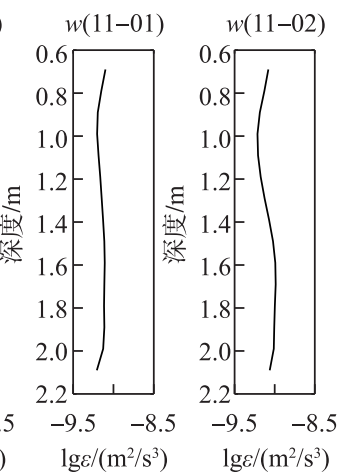

(e)

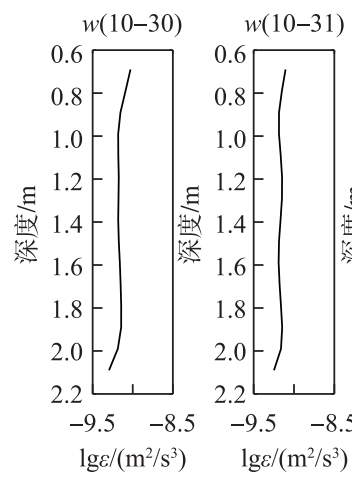

图 6 观测期间三维 $\varepsilon$ 的廓线分布: (a) 时间平均的 $\varepsilon$ 廓线分布; (b) 净热量通量对 $\varepsilon$ 的影响; (c) $u$ 方向 的 $\varepsilon$ 随时间的变化; (d) $v$ 方向 的 $\varepsilon$ 随时间的变化; (e) $w$ 方向 的 $\varepsilon$ 随时间的变化 Fig.6 The vertical profiles of the 3D $\varepsilon$ during the observation period: (a) Time-averaged $\varepsilon$ profiles;

(b) Influence of net heat flux on $\varepsilon$ profiles; (c) $\varepsilon$ change of $u$ with date;

(d) $\varepsilon$ change of $v$ with date; (e) $\varepsilon$ change of $w$ with date

区段的温差小于 $0.1^{\circ} \mathrm{C}$ (图 7). 由此也证实了风浪直接作用层中水体混合程度高、由温差引起的对流很弱, 其对 $\varepsilon$ 的影响基本可以忽略. 另一方面,鉴于风速逐渐降低,4 天中 10 月 30 日风速最大,可能是由于观测站 点离岸相对较近, 东北向的风场对湍流产生的作用可能被弱化, 造成 4 天的风浪作用层深度差别甚微. 因为 利用 ADCP 对水体三维流速进行高频观测的要求高,使得此类高频观测持续时间相对较短, 从而导致难以 定量确定风向对 $\varepsilon$ 的影响. 总之, 风浪直接作用层中风浪对 $\varepsilon$ 的直接作用突出, 温差小且其对 $\varepsilon$ 的影响效 
应弱.

10 月 30 日、31 日,水柱基本混合均匀（图 7)， $u 、 v 、 w$ 方向的常数层的起始深度基本维持在 $1 \mathrm{~m}$ 左 右. 11 月 1 日、 2 日常数层中的 $\varepsilon$ 不仅大于前两天, 更重要的是 $\varepsilon$ 在风浪直接作用层与常数层过渡区有 一个明显的向左弯曲, 使得常数层起始深度呈现下 降的趋势; 11 月 1 日、2 日存在显著的水体分层; 且 分层深度一直延伸到 $1.0 \sim 1.2 \mathrm{~m}$ (图 6), 因而温差明 显的区域在 $1.2 \mathrm{~m}$ 以下; 同时从侧面证实了 10 月 30 日、31 日朗缪尔环流产生的湍流作用深度可能伸及 $1.9 \mathrm{~m}$.

在常数层中,与 $\varepsilon$ 平衡的湍流产生不仅来源于 朗缪尔环流引发的对流，同时还来源于热力作用 尽管热力作用有促进对流和抑制对流两种作用. 就 日平均而言, 4 天内的热力通量相差无几 (图 3 ), 但 风速减弱却很明显(图 2a), 有效波高降低(图 5 )。 另一方面,此 4 天的 $L$ 也很弱（图 8)，除了 10 月 30 、 31 日少数时段外基本均小于 1 , 随时间推移,波浪、 流速切变和朗缪尔环流等动力作用持续减弱, 尤其 是 11 月 $1 、 2$ 日, $L$ 基本接近 0 ,也就是说动力效应远 小于热力作用. 因而导致朗缪尔环流引发的湍流逐 渐减弱, 这种次生环流的浴旋直径减小 ${ }^{[35]}$, 朗缪尔 环流造成或输送的湍流作用深度变浅, 导致白天热 力分层出现 (图 7). 该区段的水体势能相应增大, 正由于晚上水体散失能量导致的对流造成常数层 下部 $\varepsilon$ 增大 ; 弱化了底边界层 $\varepsilon$ 随深度递减的程度， 同时也说明在 11 月 1 日、2 日的热力作用对 $\varepsilon$ 的影 响明显大于朗缪尔环流引发的湍流. 可见在 $\varepsilon$ 的常 数层中, 朗缪尔环流和热力作用引起的对流是维持 $\varepsilon$ 稳定状态的关键因素, 其中朗缪尔环流是影响水 体分层的关键过程; 朗缪尔环流与热力耦合导致的 分层、混合的转换是引起 $\varepsilon$ 增大及其起始高度下移 的关键.

为进一步证实水温的垂直结构对 $\varepsilon$ 的影响差 异,利用各层 $\varepsilon$ 和对应深度的温差时间序列的相关 性(图 9) 分析二者之间的关系. $u 、 v 、 w$ 方向上分别 对应 1.1、1.2 和 $1.3 \mathrm{~m}$ 以上, 水温垂直结构与 $\varepsilon$ 无显 著关系; 上述深度以下,水温的垂向温差与 $\varepsilon$ 存在 显著的相关性. 鉴于朗缪尔环流对湍流和动量的垂 向输送存在显著影响 ${ }^{[36]}$, 结合图 7 的温差分布, 可

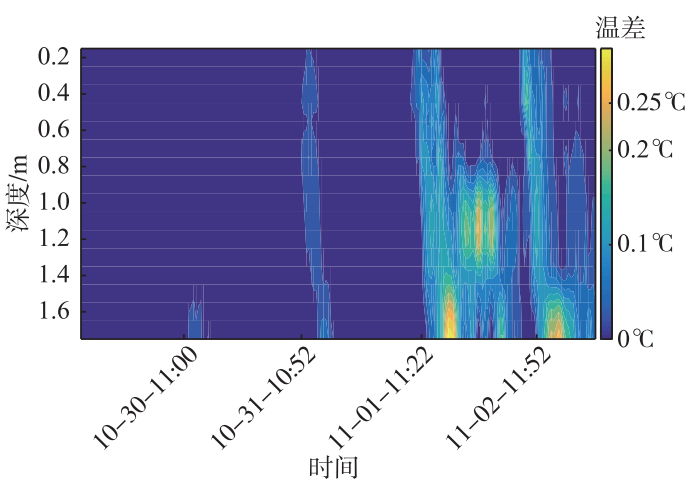

图 7 观测期间每 $10 \mathrm{~cm}$ 水柱的温差变化

Fig.7 Difference of water temperature per $10 \mathrm{~cm}$ during observation period

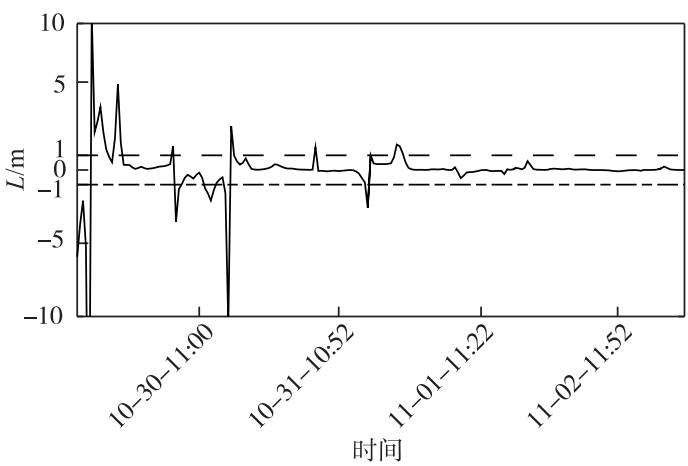

图 8 观测期间 Monin-Obukhov 长度的时间序列

Fig. 8 Time series of Monin-Obukhov length during observation period

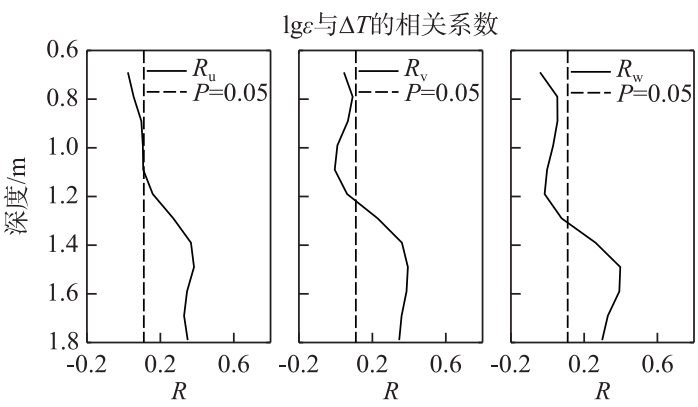

图 9 各深度温差与 $\varepsilon$ 的相关系数

Fig.9 Correlation coefficient between temperature difference and $\varepsilon$ at each depth 知朗缪尔环流影响水体热量传输和热力分层;由温差导致的湍流对 $\varepsilon$ 的贡献较为突出.

总之, 在中低风速条件下, 上表层的风浪直接作用突出, 水体混合均匀; 朗缪尔环流产生的湍流作用可 深及湖泊底边界混合层顶部, 同时其强弱也是导致热力分层与混合的关键; 热力分层的强度和位置引发的 对流混合对常数层和底部边界中的 $\varepsilon$ 贡献显著. 
鉴于 ADCP 观测所需的条件相对较高(如持续供电难以保证), 因而观测点选择有电源的中国科学院太 湖湖泊生态系统研究站的栈桥头, 导致难以定量描述风向对 $\varepsilon$ 的影响; 太湖水深较浅, 本次观测采用悬挂 式, 有效观测数据从 $0.7 \mathrm{~m}$ 的水深开始, 因而波浪破碎和水平速度的垂直切变等各因子对 $\varepsilon$ 的定量影响对湍 流动能产生率的贡献难以厘清.

观测时段风场资料是气象局观测的逐时资料, 是整点的十分钟平均风场. 观测时段的风速小于 $5.5 \mathrm{~m} / \mathrm{s}$. 为了考察观测时段的风场是否具有代表性, 统计了 2017 年东山站全年逐时风速资料, 发现全年中低于 5.5 $\mathrm{m} / \mathrm{s}$ 的风速占全年资料的 $96.96 \%$, 说明观测时段风场具有较强的代表性; 同样辐射通量的日变化也具有典 型代表性(图 3). 因此尽管观测时段短,但研究结果能较好地反映气象场对大型浅水湖泊中 $\varepsilon$ 的影响效应.

\section{4 结论}

通过高频野外观测, 揭示了太湖 $\varepsilon$ 廓线分布特征, 分析动力、热力强迫作用对太湖湍流的作用及深度分 布. 有利于探讨大型浅水湖泊日分层规律、营养盐和藻类垂向分布及水生生态系统演变.

1) 太湖几何深度浅, 其 $\varepsilon$ 依然存在典型的 3 层: 随深度递减的 $\varepsilon$ 风浪直接作用层, 深度在 $1.0 \mathrm{~m}$ 左右以 上; 基本不随深度变化的常数层, 深度区间介于 $1.0 \sim 1.9 \mathrm{~m}$ 之间; 随后是随深度递减的底边界混合层.

2) 太湖中, 水平流速切变、波浪等作用导致风浪作用层均匀混合; 热力分层的强弱 (垂向温差)、位置对 常数层和底部边界中的 $\varepsilon$ 贡献显著,甚至造成 $\varepsilon$ 的 常数层起始深度下移.

\section{5 参考文献}

[ 1 ] Qin BQ, Zhu GW, Zhang L et al. Wave effects on nutrient release of sediments from Lake Taihu by flume experiments. Science in China: Series D: Earth Sciences, 2005, 35(S2): 33-44. [ 秦伯强, 朱广伟, 张路等. 大型浅水湖泊沉积物内 源营养盐释放模式及其估算方法. 中国科学: D 辑: 地球科学, 2005, 35(S2) : 33-44.]

[ 2 ] Huang L, Fang H, He G et al. Effect of internal loading on phosphorus distribution in the Taihu Lake driven by wind waves and lake currents. Environmental Pollution, 2016, 219: 760-773. DOI: 10.1016/j.envpol.2016.07.049.

[ 3 ] Wu T, Timo H, Qin B et al. In-situ erosion of cohesive in a large shallow lake experiencing long-term decline in wind speed. Journal of Hydrology, 2016, 539: 254-264. DOI: 10.1016/j.jhydrol.2016.05.021.

[ 4 ] Zheng S, Wang P, Wang C et al. Sediment resuspension under action of wind in Taihu Lake, China. International Journal of Sediment Research, 2015, 30: 48-52. DOI: 10.1016/s1001-6279(15)60005-1.

[ 5 ] Wu T, Qin B, Zhu G et al. Modeling of turbidity dynamics caused by wind-induced waves and current in Taihu Lake. International Journal of Sediment Research, 2013, 28: 139-148. DOI: 10.1016/S1001-6279(13)60026-8.

[ 6 ] Deng J, Chen F, Liu X et al. Horizontal migration of algal patches associated with cyanobacterial blooms in an eutrophic shallow lake. Ecological Engineer, 2016, 87: 185-193. DOI: 10.1016/j.ecoleng.2015.12.017.

[ 7 ] Qin BQ, Hu WP, Chen WM et al. Studies on the hydrodynamic processes and relater factors in Meiliang Bay. J Lake Sci, 2000, 12(4) : 327-335. DOI: 10.18307/2000.0406. [ 秦伯强, 胡维平, 陈伟民等. 太湖梅梁湾水动力及其相关过程 的研究. 湖泊科学, $2000,12(4): 327-335$. ]

[ 8 ] Pang Y, Pu PM. Numerical study of wind-driven current in Taihu lake with the air-water coupling model. J Lake Sci, 1996, 8 (2) : 97-102. DOI: 10.18307/1996.0201. [ 逢勇, 涭培民. 大气-水耦合模式下三维太湖糊流场研究. 湖泊科 学, 1996, 8(2): 97-102.]

[ 9 ] Hu WP, Pu PM, Qin BQ. A three-dimensional numerical simulation on the dynamics in Taihu lake, China( 1): the water level and the current during the 9711 typhoon process. J Lake Sci, 1998, 10(2) : 17-25. DOI: 10.18307/1998.0403. [胡 维平, 誉培民, 秦伯强. 太湖水动力学三维数值试验研究—1. 风生流和风涌增减水的三维数值模拟. 湖泊科学, $1998, \mathbf{1 0}(2): 17-25$.]

[10] Xu XF, Liu QQ. Numerical study on the characteristics of wind-induced current in Taihu Lake. Chinese Journal of Hydrodynamics, 2009, 24(4): 512-518. [许旭峰, 刘青泉. 太湖风生流特征的数值模拟研究. 水动力学研究与进展, $2009, \mathbf{2 4}(4): 512-518$. ]

[11] Belcher E, Grant M, Hanley E et al. A global perspective on Langmuir turbulence in the ocean surface boundary layer. Geophysical Research Letters, 2012, 39(18) : 9. DOI: 10.1029/2012GL052932. 
[12] Brainerd KE, Gregg MC. Diurnal restratification and turbulence in the oceanic surface mixed layer: 1. Observations. Journal of Geophysical Research Oceans, 1993, 98(C12) : 22645-22656. DOI: 10.1029/93JC02297.

[13] Baumert HZ, Simpson J, Sündermann J et al. Marine turbulence: theories, observations, and models. Cambridge: Cambridge University Press, 2005: 115-126.

[14] Wu H, Patterson GK, Doornm V. Distribution of turbulence energy dissipation rates in a Rushton turbine stirredmixer. Experiments in Fluids, 1989, 8(3/4) : 153-160. DOI: 10.1007/BF00195789

[15] Sreenivasan KR. On the universality of the Kolmogorov constant. Physics of Fluids, 1995, 7(11) : 2778-2784. DOI: 10. 1063/1.868656.

[16] Doron P, Bertuccioli L, Katz J et al. Turbulence characteristics and dissipation estimates in the coastal ocean bottomboundary layer from PIV data. Journal of Physical Oceanography, 2001, 31( 8 ) : 2108-2134. DOI: 10.1175/1520-0485(2001) $031<2108$ : TC- ADEI $>2.0 . \mathrm{CO} ; 2$.

[17] Bluteau CE, Jones NL, Ivey GN. Estimating turbulent kinetic energy dissipation using the inertial subrangemethod in environmental flows. Limnology and Oceanography Methods, 2011, 9(7) : 302-321. DOI: 10.4319/lom.2011.9.302.

[18] Callaghan AH, Ward B, Vialard J. Influence of surface forcing on near-surface andmixing layer turbulence in the tropical Indian Ocean. Deep Sea Research Part I: Oceanographic Research Papers, 2014, 94: 107-123. DOI : 10.1016/j.dsr.2014. 08.009.

[19] Caldwell DR, Lien RC, Moum JN et al. Turbulence decay and restratification in the equatorial ocean surface layer following nighttime convection. Journal of Physical Oceanography, 1997, 27(1997) : 1120-1132. DOI: 10.1175/ 1520-0485 (1997) 0272. 0. CO;2.

[20] Liu ZY, Wei H. Estimation to the turbulent kinetic energy dissipation rate and bottom shear stress in the tidal bottom boundary layer of the Yellow Sea. Progress in Natural Science, 2007, 17(3) : 362-369. DOI: 10.1080/10020070612331343260. [刘] 志宇, 魏皓. 黄海潮流底边界层内湍动能耗散率与底应力的估计. 自然科学进展, 2007, 17(3): 362-369.]

[21] Qin BQ, Yang GJ, Ma JR. Dynamics of variability and mechanism of harmful cyanobacteria bloom in Lake Taihu, China. Chinese Science Bulletin, 2016, 61(7) : 759-770. DOI: 10.1360/N972015-00400. [秦伯强, 杨桂军, 马健荣等. 太湖 蓝藻水华“暴发”的动态特征及其机制. 科学通报, 2016, 61(7): 759-770.]

[22] Zhou J, Qin B, Han X et al. Turbulence increases the risk ofmicrocystin exposure in a eutrophic lake (Lake Taihu) during cyanobacterial bloom periods. Harmful Algae, 2016, 55: 213-220. DOI: 10.1016/j.hal.2016.03.016.

[23] Xie P, Xia J, Dou M et al. Research into the effects of the middle route of China's south-to-north water transfer project on water bloom in the middle-down stream of Hanjiang River and the countermeasures Part I: An analysis of the key factors generating water bloom in Hanjiang River. Journal of Natural Resources, 2004, 19(4) : 418-423. DOI: 10.11849/zrzyxb. 2004.04.002. [ 谢平, 夏军, 窦明等. 南水北调中线工程对汉江中下游水华的影响及对策研究 ( I ) 一一汉江水华发 生的关键因子分析. 自然资源学报, 2004, 19(4): 418-423.]

[24] Huisman J, Arrayãs M, Ebert U et al. How do sinking phytoplankton speciesmanage to persist? American Naturalist, 2002, 159(3) : 245-254. DOI: 10.1086/338511.

[25] Goring DG, Nikora VI. Despikingacoustic dopplervelocimeter data. Journal of Hydraulic Engineering, 2002, 128( 1 ): 117-126. DOI: 10.1061/( ASCE) 0733-9429(2002)128:1(117).

[26] Singh SK, Debnath K. Combined effects of wave and current in free surface turbulent flow. Ocean Engineering, 2016, 127: 170-189. DOI: 10.1016/j.oceaneng.2016.10.014

[27] Maggiore A, Zavatarelli M, Angelucci MG et al. Surface heat and water fluxes in the Adriatic Sea: seasonal and interannual variability. Physics and Chemistry of the Earth, 1998, 23(5/6) : 561-567. DOI: 10.1016/s0079-1946(98)00070-6.

[28] Churchill JH, Kerfoot WC. The impact of surface heat flux and wind on thermal stratification in portage lake, Michigan. Journal of Great Lakes Research, 2007, 33(1) : 143-155. DOI: 10.3394/0380-1330(2007)33[143:tioshf]2.0.co;2.

[29] Kim TW, Cho YK. Calculation of heat flux in amacrotidal flat using FVCOM. Journal of Geophysical Research Oceans, 2011, 116(C3). DOI: 10.1029/2010JC006568.

[30] Zhao Q, Sun J, Zhu G. Simulation and exploration of themechanism underlying the spatiotemporal distribution of surfacemixed layer depth in a large shallow lake. Advances in Atmospheric Science, 2012, 29(6) : 1360-1373.

[31] Gemmrich JR, Farmer DM. Near-Surface turbulence in the presence of breaking waves. Journal of Physical Oceanography, 2004, 34(5) : 1067-1086. DOI: 10.1175/1520-0485(2004) 034<1067 : NTITPO>2.0.CO;2. 
[32] Burchard H, Craig PD, Gemmrich JR et al. Observational and numericalmodelingmethods for quantifying coastal ocean turbulence andmixing. Progress in Oceanography, 2008, 76 (4) : 399-442. DOI: 10.1016/j.pocean.2007.09.005.

[33] Thorpe SA, Osborn TR, Jackson JFE et al. Measurements of turbulence in the upper-ocean mixing layer using autosub. Journal of Physical Oceanography, 2003, 33(1) : 122-145. DOI: 10.1175/1520-0485(2003)033<0122: MOTITU>2.0. $\mathrm{CO} ; 2$.

[34] Polton JA, Belcher SE. Langmuir turbulence and deeply penetrating jets in an unstratified mixed layer. Journal of Geophysical Research, 2007, 112( C9) : C09020. DOI: 10.1029/2007jc004205.

[35] Mazumder BS, Ojha SP. Turbulence statistics of flow due to wave-current interaction. Flow Measurement \& Instrumentation, 2007, 18(3/4) : 129-138. DOI: 10.1016/j.flowmeasinst.2007.05.001.

[36] Araujo M, Dartus D, Maurel P et al. Langmuir circulations and enhanced turbulence beneath wind-waves. Ocean Modelling, 2001, 3(1/2) : 109-126. DOI: 10.1016/s1463-5003(01) 00004-x. 